\title{
Stress Redistribution of Concrete Prisms Due to Creep and Shrinkage: Long- Term Observations and Analysis
}

\author{
P. Criel ${ }^{1}$; R. Caspeele ${ }^{1}$; N. Reybrouck ${ }^{1}$; S. Matthys ${ }^{1}$; and L. Taerwe ${ }^{1}$ \\ ${ }^{1}$ Magnel Laboratory for Concrete Research, Department of Structural Engineering, \\ Ghent University, Ghent, Belgium. E-mail: Pieterjan.Criel@UGent.be
}

\begin{abstract}
In 1979, 16 concrete prisms with dimensions 140x150x4000 mm were casted in the Magnel Laboratory for Concrete Research. These prisms differ by the amount of passive reinforcement and by the applied loading level. Four reinforcement ratios were considered, i.e. $0 \%, 1.5 \%, 3 \%$ and $6 \%$. For each reinforcement ratio a prism was subjected to an axial load corresponding to a concrete stress of $0,5,10$ or 15 $\mathrm{MPa}$. The combination of both parameters results in a total of 16 specimens. The compressive stress was applied to the prisms by means of post-tensioned unbonded strands at an age of 28 days. The stress level was kept constant during the first 12 years of the experiment by re-adjusting the force in the strands when the deviation exceeded $2 \%$ of the initial value. Afterwards, no re-adjusting of the strands took place. Significant redistribution of the stresses between the concrete and the steel can be expected due to the creep and shrinkage of concrete. This redistribution is larger for the prisms with a higher reinforcement ratio, resulting in lower creep and shrinkage strains. The time-dependent response of these prisms was modelled taking into account the prestress losses and stress redistribution between the concrete and the steel. For reasons of numerical efficiency, the compliance function was approximated by a Dirichtlet series using continuous retardation spectra. A comparison between the predicted results and the measurements is given for two prisms.
\end{abstract}

\section{INTRODUCTION}

Creep and shrinkage are important time-dependent phenomena in reinforced concrete structures. Although consensus on the details of the different mechanisms responsible for the time-dependent behaviour is not yet reached (Koenders et al., 2009; Yue, 1992), the effects of creep and shrinkage are well known and many reliable material models are available in literature. A large part of this research is the result of experiments on plain concrete without any reinforcing steel present and under constant loading conditions. However, in reality the load on concrete elements is rarely constant in function of time and reinforcement steel is mostly present. In order to determine the influence of reinforcing steel on the time-dependent deformations of concrete a series of 16 prisms with dimensions $140 \times 150 \times 4000 \mathrm{~mm}^{3}$ were manufactured at the Magnel Laboratory for Concrete Research (Lambotte et al., 1986, 1988). These prisms differ by the amount of passive reinforcement respectively $0,1.5,3$ and $6 \%$ of the concrete section - and by the applied level of 
axial stress - respectively $0,5,10$ and $15 \mathrm{MPa}$. One prism for each combination was tested. An overview of the experimental programme is given in Figure 1.

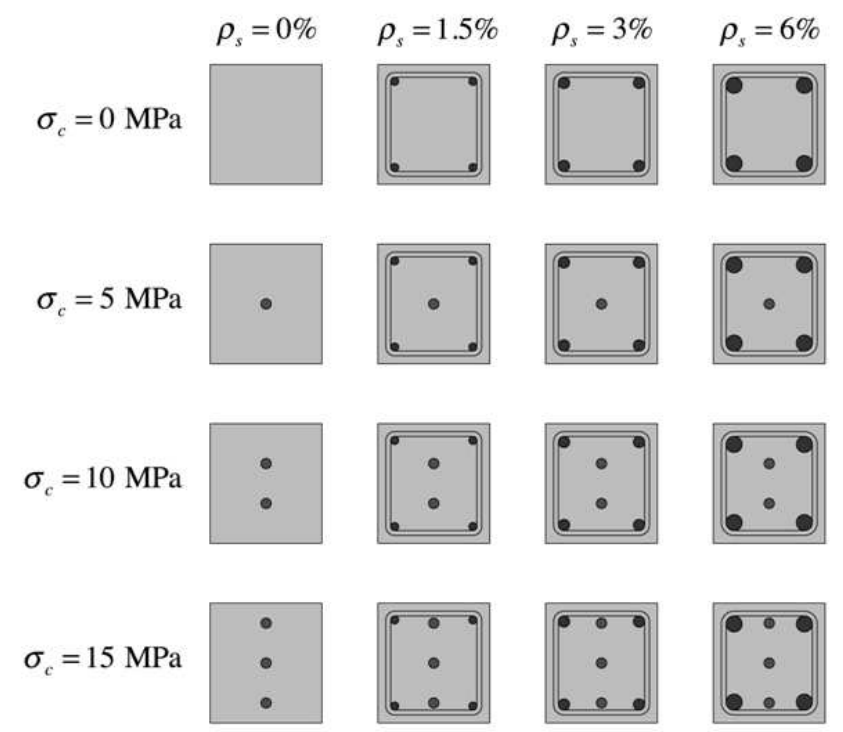

Figure 1 Reinforcing bars and unbounded strands arrangements for the 16 combinations of passive reinforcement ratios and applied stress levels.

Each prism is designated with a prefix "W" followed by the percentage of passive reinforcing steel, a dash and the level of applied stress; e.g. prism W0-0 is the prism in the top-left position in Figure 1, containing $0 \%$ reinforcing steel and zero applied stress.

The compatibility of the longitudinal deformations has as a consequence that the creep and shrinkage of the prisms are partly restrained by the reinforcement steel. This results in a redistribution between the stress in the concrete, which will decrease, and the stress in the reinforcement steel, which will increase in order for the crosssection to remain in longitudinal equilibrium. In the scope of this paper it is assumed that the bond between the concrete and the reinforcement steel is perfect.

\section{TEST PROCEDURE}

One day after casting, each prism was placed in a frame on two supports, of which one support allows for horizontal movements. The distance between the two supports was determined in such a way that the bending moments above the supports and in the mid-section due to the self-weight would have the same (minimal) value.

The prisms are axially prestressed with unbonded strands at 28 days. The strands were placed in a prestressing bed 96 hours before anchorage to reduce longterm relaxation losses. The prestressing force of each strand was measured using a load cell placed between the bearing plate attached to the endpoints of the prisms and the strand anchorage. An illustration of the test setup for each of the prims is given in Figure 2. 


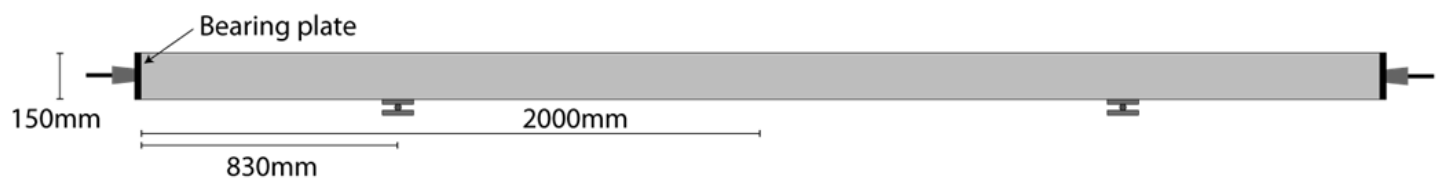

Figure 2 Test setup of the prisms

The axial force in the strands $P_{0}$ is determined in such a way that the resulting stress in the concrete is equal to respectively $0,5,10$ or $15 \mathrm{MPa}$ for the specified cases according to

$$
P_{0}=\sigma_{c}\left(A_{c}+\alpha A_{S}\right)
$$

where $A_{c}$ is the area of the concrete section, $A_{s}$ is the area of the passive reinforcement, $\sigma_{c}$ is the required concrete stress and $\alpha$ is the ratio between the moduli of elasticity of concrete and steel respectively. For $\alpha$ a theoretical value of 6 was assumed.

Each time the prestress losses reached $2 \%$ of the initial prestressing force, the force in the tendons was adjusted to 1.02 times the initial value. In this manner the external compressive force was kept approximately constant during the experiment.

\section{MATERIALS}

A traditional concrete with a water-cement ratio equal to 0.46 was used. The composition of the mixture in given in Table 1.

Table 1. Concrete mixture.

\begin{tabular}{cc}
\hline Component & $\mathrm{kg} / \mathrm{m}^{3}$ \\
\hline Cement CEM I 52.5 N & 360 \\
Gravel 8/16 & 1060 \\
Gravel 4/8 & 190 \\
Sand 2/5 & 100 \\
Sand 0/2 & 535 \\
Water & 165 \\
\hline
\end{tabular}

The mean compressive strength (cubes with side lengths $158 \mathrm{~mm}$ ) at 28 days was equal to $47.4 \mathrm{MPa}$.

The unbonded tendons have a nominal diameter of $12.7 \mathrm{~mm}$ and have a very low relaxation. There are respectively $0,1,2$ and 3 tendons used for the considered stress levels in the prisms

High-bond reinforcement bars with diameters 10, 14 and $20 \mathrm{~mm}$ were used for the passive reinforcement. 


\section{MEASUREMENT RESULTS}

Nearly all reinforced prisms developed small cracks with a maximum crack width of $0.02 \mathrm{~mm}$ during the first 28 days of the experiment (when no axial loading was applied). These cracks can be attributed to the restrained shrinkage of the concrete.
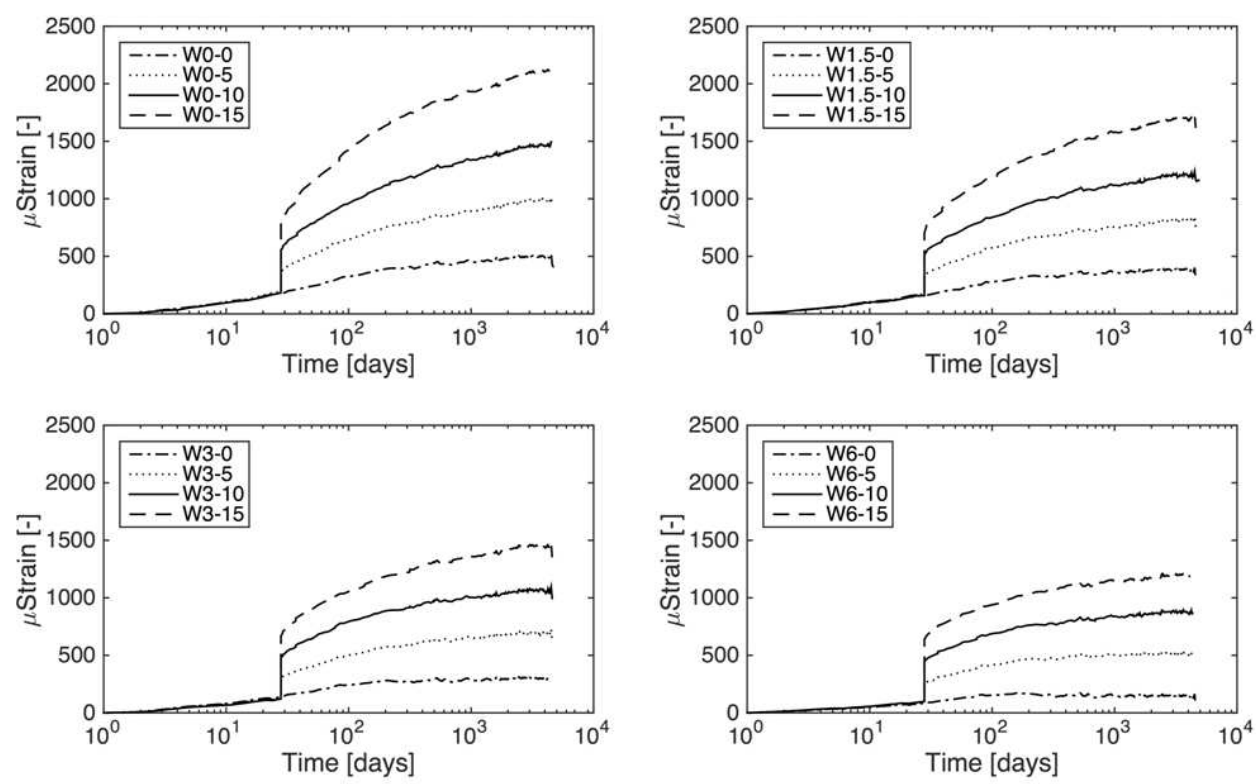

Figure 3 Measurement results of the axial deformation of the prisms.

The strain measurements of the prisms are given in Figure 3 up to 4500 days (12 years). It is observed that the measured concrete strain is proportional to the applied stress for the different reinforcement ratios.

Comparing W0-0 till W6-0, it is noted that the strain for the prisms with passive reinforcement steel is significantly less than the strain measured in the prism where no steel is present. This difference is attributed to the passive reinforcing steel, which limits the free shrinkage of the concrete.

It is noted that the strain of prism W6-0 shows a constant value starting from approximately 200 days. The concrete shrinkage continues but the total deformation does not increase any further. Due to the high percentage of passive reinforcement, tensile stresses are introduced in the concrete and the concrete cracked. This is visually confirmed by the many cracks in this particular prism.

\section{NUMERICAL PROCEDURE}

\section{Creep strain}

The uniaxial constitutive relation defining concrete as an ageing viscoelastic material is given by (Bažant, 1988): 


$$
\varepsilon(t)=\int_{0}^{t} J\left(t, t^{\prime}\right) d \sigma\left(t^{\prime}\right)+\varepsilon_{s h}(t)
$$

in which $t$ is the time of interest, $t^{\prime}$ is the time of application of the concrete stress, $\sigma$ is the concrete stress,$\varepsilon(t)$ is the total concrete strain, $\varepsilon_{s h}(t)$ is the concrete shrinkage strain.

$J\left(t, t^{\prime}\right)$ is the creep compliance function. This integral is known as a Stieltjes integral. There is no compliance function $J\left(t, t^{\prime}\right)$ which is sufficiently accurate for this integral to be solvable in an analytically closed form. A numerical solution of this integral is possible, but this requires that the complete stress history is kept in memory for each new time-step (Gilbert \& Ranzi, 2010). This is only feasible for simple problems.

In order to make the calculation of the Stieltjes integral more feasible, the creep compliance function can be approximated by a series of exponential functions with real exponents, so called Dirichlet series (Bažant, 1975, 1988). Hence, the compliance function $J\left(t, t^{\prime}\right)$ can be rewritten as:

$$
J\left(t, t^{\prime}\right)=\frac{1}{E\left(t^{\prime}\right)}+\sum_{\mu=1}^{m} \frac{1}{\widehat{E}_{\mu}}\left(1-e^{-\left(t-t^{\prime}\right) / \tau_{\mu}}\right)
$$

in which $\tau_{\mu}$ are the chosen retardation times, $E\left(t^{\prime}\right)$ is the modulus of elasticity of the concrete at time $t^{\prime}$ and $\hat{E}_{\mu}$ are coefficients which depend on the age of load application $t^{\prime}$. The coefficients $\hat{E}_{\mu}$ have the same dimension as the modulus of elasticity and must be determined in such a way that the error between the theoretical creep compliance function and the series approximation is minimal. Notice that the Dirichtlet series is written in function of the time lag $t-t^{\prime}$ for a given application time $t^{\prime}$. To determine the coefficients $\widehat{E}_{\mu}$ it is most convenient to first select a number of $t^{\prime}$ values and fit $J\left(t, t^{\prime}\right)$ as a function of the lag time $t-t^{\prime}$ for each fixed $t^{\prime}$. Since the concrete stress gradually decreases, a Dirichtlet series was defined for each time step in the analysis. A uniform distribution of the time-step in log-time recommended choice (Jendele \& Phillips, 1992).

Fitting a Dirichlet series on a function is not as straightforward as a Fourier series (Bažant, 1973). The coefficients $\widehat{E}_{\mu}$ should be regarded as a discrete spectrum of the Dirichtlet series for given retardation times $\tau_{\mu}$. If the Dirichlet series is composed out of an infinite number of terms, each with a coefficient $\widehat{E}_{\mu}$ and a retardation time $\tau_{\mu}$, a continuous function $L(\tau)$ is created. This function $L(\tau)$ can be regarded as the continuous spectrum of a Dirichtlet series with an infinite number of terms (Jirásek \& Havlásek, 2014).

Jirásek \& Havlásek (2014) showed that an approximation of $L(\tau)$ can be obtained analytically be using the Post-Widdler formula 


$$
L(\tau)_{(k)}=-\frac{(-k \tau)^{k}}{(k-1) !} \varphi^{(k)}(k \tau)
$$

It was shown that a sequence of approximations converges to the continuous spectrum $L(\tau)$, in which $\varphi(\cdot)$ is the creep function, $\varphi^{(k)}(\cdot)$ is the $k^{\text {th }}$ derivative of the creep function and $k$ is the order of approximation.

Values for the coefficients $\hat{E}_{\mu}$ can be determined by integration of $L(\tau)$. With these coefficient an approximated compliance function $J\left(t, t^{\prime}\right)$ is constructed which allows for an analytical solution of the Stieltjes integral.

\section{Stress redistribution}

the strain of the steel and the strain of the concrete should be equal at each time $t$ :

$$
\varepsilon_{c}(t)=\varepsilon_{S}(t)=\varepsilon(t)
$$

The steel stress can be written as:

$$
\sigma_{s}(t)=E_{s} \varepsilon(t)
$$

where $E_{s}$ is the modulus of elasticity of the reinforcement steel. The longitudinal equilibrium of the prisms can be written as:

$$
P(t)=A_{s} \sigma_{s}(t)+A_{c} \sigma_{c}(t)
$$

The concrete stress which would induce by a strain $\Delta \varepsilon$ is given by:

$$
\sigma_{c}=\Delta \varepsilon E_{c}
$$

The corresponding force on the concrete area $A_{c}$ is given by:

$$
F=\sigma_{c} A_{c}=\Delta \varepsilon E_{c} A_{c}
$$

If the same force is exerted on a concrete section that contains passive reinforcement the following equality can be written:

$$
\Delta \varepsilon E_{c} A_{c}=\Delta \varepsilon_{c o r r}\left(E_{c} A_{c}+E_{s} A_{s}\right)
$$

with $A_{s}$ the area of the steel section and $\Delta \varepsilon_{\text {corr }}$ the strain of the section containing both concrete and reinforcement steel.

The ratio between the corrected strain $\Delta \varepsilon_{\text {corr }}$ and the assumed free strain of $\Delta \varepsilon$ is given by:

$$
\frac{\Delta \varepsilon_{\text {corr }}}{\Delta \varepsilon}=\frac{E_{c} A_{c}}{E_{c} A_{c}+E_{s} A_{s}}
$$


The same reasoning can be applied to shrinkage strains as well as creep strains, with the difference that the creep strain will depend on the concrete stress in the considered time-step, while the shrinkage strain is independent of the stresses in the concrete.

\section{Prestress losses}

Due to the concrete creep and shrinkage the unbonded tendons will lose part of their prestressing force. The equivalent lost prestress force for a given strain $\Delta \varepsilon_{\text {corr }}$ is given by:

$$
\Delta P=\Delta \varepsilon_{c o r r} A_{p} E_{p}
$$

where $A_{p}$ is the area of the prestressing tendons and $E_{p}$ is the modulus of elasticity of the prestressing steel.

\section{General procedure}

As in (Taerwe, 1990), also in this paper the creep and shrinkage models suggested by Model Code 90 are applied (fib, 1993).

A series of times $t_{1}, t_{2}, \ldots, t_{r}, \ldots, t_{200}$ is chosen, distributed uniformly on a log-scale. A total of 200 calculation steps were considered in the analysis. Since the nature of this problem results in a continuous decreasing concrete stress, the Dirichlet series to approximate the compliance function needs to be determined for each time $t_{r}$ in the time series.

During the first 28 days no prestressing is applied on the prisms. The prisms will shrink during this period, introducing tensile stresses in the concrete due to the restraining effect. After 28 days the prestressing force is applied. Starting from this point the prisms will start to creep. To avoid erroneous predictions of the instantaneous response of the prisms, the instantaneous response is calculated by considering a very short time step after 28 days. In each time step the creep and shrinkage strains of the concrete are corrected as mentioned above taking into account the restraining effect of the reinforcement steel. With this correction, the stresses in the reinforcing steel and in the concrete are adjusted so that an equilibrium is reached with the prestressing force $P(t)$.

\section{RESULTS}

The procedure described above is applied for prism W1.5-10 of the experimental test programme. The results of the calculation is given in Figure 4. 

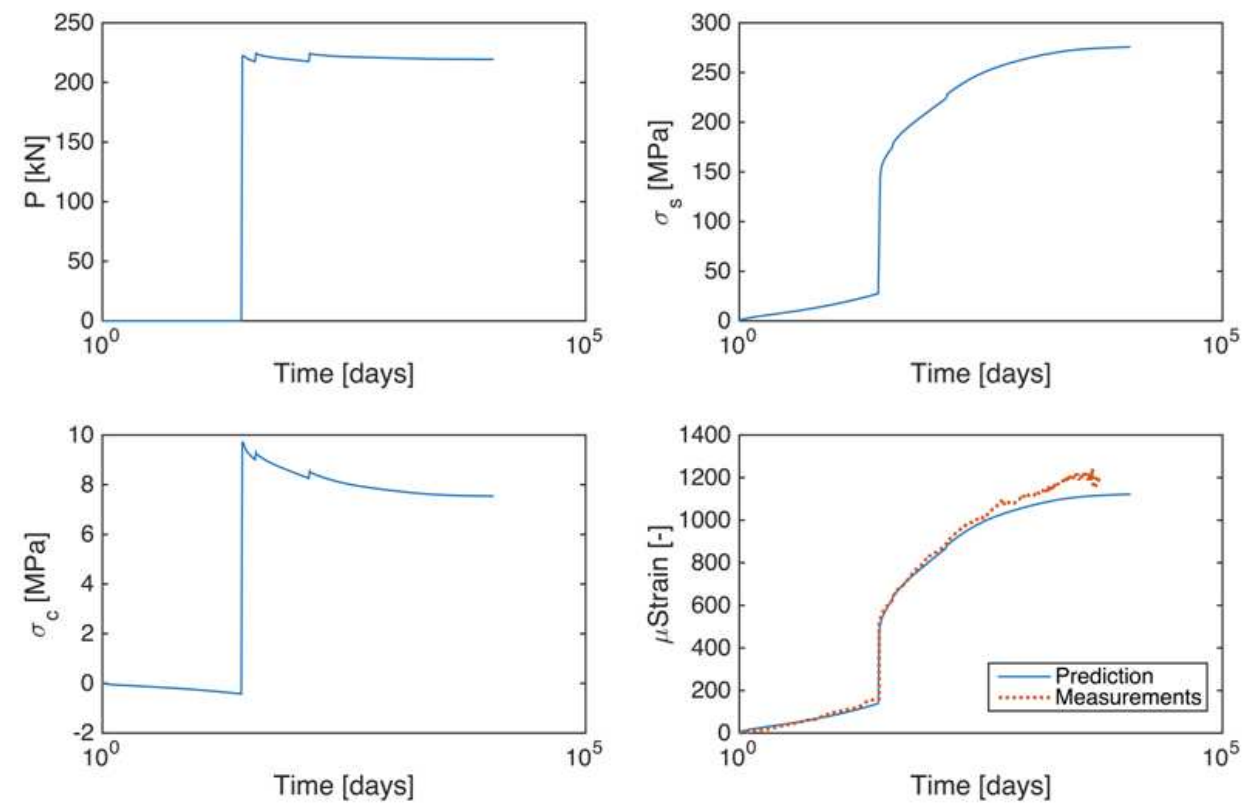

Figure 4 Results of the calculation for prism W1.5-10. (a) The prestressing force (b) The stress in the reinforcement (c) The stress in the concrete (d) The predicted and measured deformation of the prism

In the period before the application of the load the model shows a build-up of tensile stress in the concrete, as shown in Figure 4 (c). This is due to the restrained shrinkage. The load is applied at 28 days. The predicted deformation shows good agreement with the measurements. The concrete stress decreases over time starting from a pre-defined stress equal to $10 \mathrm{MPa}$ and drops to $7.5 \mathrm{MPa}$. The stress in the reinforcement steel is almost doubled compared to the stress immediately after the application of the load.

\section{CONCLUSIONS}

From the available experiments and the numerical analysis the following conclusions can be drawn.

- Measurements of axial deformations of concrete prisms subjected to a quasisustained load during 12 years are presented. The experiments show that the time-dependent deformations of reinforced prisms are strongly affected by the presence of passive reinforcement steel. This is due to the significant redistribution of the stresses in the concrete section.

- An approximation of the creep compliance function by a Dirichtlet series allows for the Stieltjes integral to be solvable in an analytically closed form. Additionally, the load history does not need to be stored in order to calculate the deformation in a next time-step.

- The predicted axial deformations taking into account the stress redistribution and the prestress losses show good agreement with the measurements. 


\section{ACKNOWLEDGEMENTS}

The authors would like to thank the Agency for Innovation by Science and Technology in Flanders (IWT) and the Buildsoft company for supporting this research.

\section{REFERENCES}

Bažant, Z. P. (1973). Dirichlet Series Creep Function for Aging Concrete Journal of the Engineering Mechanics Division, 99(2), 367-387.

Bažant, Z. P. (1975). I. - Theory of Creep and Shrinkage in Concrete Structures: A Précis of Recent Developments. In S. Nemat-Nasser (Ed.), Mechanics Today (pp. 1-93): Pergamon.

Bažant, Z. P. (1988). Mathematical Modeling of Creep and Shrinkage of Concrete. New York: John Wiley \& Sons.

fib. (1993). CEB-FIP Model Code 90. London: Thomas Telford

Gilbert, R. I., \& Ranzi, G. (2010). Time-Dependent Behaviour of Concrete Structures: Taylor \& Francis.

Jendele, L., \& Phillips, D. V. (1992). Finite element software for creep and shrinkage in concrete. Computers \&amp; Structures, 45(1), 113-126. doi: 10.1016/00457949(92)90349-5

Jirásek, M., \& Havlásek, P. (2014). Accurate approximations of concrete creep compliance functions based on continuous retardation spectra. Computers \& Structures, 135(0), 155-168. doi:

http://dx.doi.org/10.1016/j.compstruc.2014.01.024

Koenders, E. A. B., van der Ham, H. W. M., van Breugel, K., \& Lokhorst, S. J. (2009). Modelling creep by microstructural changes. Paper presented at the Concreep 8, Ise-Shima, Japan.

Lambotte, H., Van Nieuwenburg, D., \& Cocquyt, F. (1986). Influence of NonPrestressed Steel on Shrinkage and Creep deformations and on Steel-Concrete Stress Redistribution. In M. Z. Cohn (Ed.), Partial Prestressing, From Theory to Practice (NATO ASI Series E: Applied Sciences, V. 2) (pp. 45-56). Doordrecht: Martinus Nijhoff Publishers.

Lambotte, H., Van Nieuwenburg, D., \& Taerwe, L. (1988). Cracking and Crack Widths of Partially Prestressed Concrete Members. Paper presented at the European Conference on Cracking of Concrete and Durability of Constructions, AFREM-CCE, Saint-Remy-Les-Cheveuse.

Taerwe, L. (1990). Simplified Analysis of Stress Redistribution in Partially Prestressed Concrete Sections. Aci Structural Journal, 87(11), 93-98.

Yue, L. L. (1992). Creep Recovery of Plain Concrete under Uniaxial Compression. $\mathrm{PhD}$ thesis, Ghent University, Ghent. 